can be traced, at least in Sheffield. The situation may be different in larger cities such as London, for example. Only two patients from the sample had moved outside the local area.

No patients were homeless at follow-up. This finding may suggest some caution is needed in interpreting studies that suggest a high level of mental illness among the homeless as implying that the rundown of the psychiatric hospital should be slowed. A poorly reasoned polemic from Weller (1989) can find its way into the prestigious columns of Nature, revealing the current bias against deinstitutionalisation in Britain. The present study gives some picture of what actually happens to patients after discharge.

It is important to note the high rate of death among this patient group. The long-stay population in psychiatric hospitals is elderly. The rehabilitation and long-stay service in Sheffield at follow-up eight years later has a slightly increased average age. If there are no further admissions a significant attrition rate through death can continue to be expected. It is important to remember that the decline in the number of patients in psychiatric hospitals is not only due to discharge but to death expected because of the age distribution of the longstay population.

Of those discharged from hospital the majority were still living in fairly institutional surroundings. A significant proportion of this care in Sheffield is provided in private residential homes for the mentally ill. The person who was in prison was there for attempted murder and was not regarded as mentally ill.
The quality of life issue for those out of hospital compared with those remaining in hospital has not been answered by this study. Nor could it easily be answered because of the difficulty measuring quality of life, obtaining adequate control groups, and the lack of randomisation. Those who are resettled are selected to some extent, making the interpretation of any comparison with those remaining in hospital difficult.

If problems are being caused by the rundown of the psychiatric hospital, it may be because of the limited facilities for the new long-stay, rather than because of the resettlement of the older long-stay patients. Any argument about community care and the preservation of the traditional psychiatric hospital should distinguish between the needs of people who can no longer get into the psychiatric system, as opposed to the needs of the people who may just have been thrown out. However, a substantial proportion of the rehabilitation and long-stay wards in this study at follow-up were occupied by newly admitted patients over the eight years since the census.

Government policy is moving in the direction of smaller units for continuous care of psychiatric patients. The relatively small group of patients placed outside hospital over eight years could suggest there is scope for more, not less, resettlement of patients from the traditional psychiatric hospital.

\section{Reference}

WeLLER, M. P. I (1989) Mental illness - who cares? Nature, $339,249-252$

\title{
Absconders from a district general hospital
}

\section{B. T. FARID, Consultant Psychiatrist, New Cross Hospital, Wolverhampton WV10 0QP}

The patient absconding without leave (AWOL) produces considerable anxiety to staff, relatives and the public, and involves a great deal of staff time in notification, search and enquiry to the detriment of the care of other patients. Incidents of AWOL are potentially dangerous and all efforts to predict these incidents and to deal with them appropriately should be made.

\section{The study \\ Aims}

(a) To find out the pattern of AWOL incidents at New Cross Hospital Psychiatric Unit including characteristics of the patients and any possible variables that will assist in predicting them. 
(b) To compare the local pattern of AWOL to other avarilable data.

(c) To ascertain whether the level of recording alt these incidents is satisfactory.

(d) To ascertain whether the procedure of dealing with $A W O L$ is adequate and whether any changes should be made.

A list of patients who have ábsconded from the psychiatric wards over three months from 1 Febritary to 30 April 1990 was obtained from the Support Services Manager. Medical and nursing notes relating to each of these incidents were obtained. A list of relevant variables was recorded from the notes. The level of nursing staff at the time of each incident was obtained from the Patiènt Care Manager. The data were analysed and compared to the ondy published paper on the subject (Tomison, 1989).

\section{Findings}

Some of the dates, names and hospital numbers provided were not accuräte and some incidents were not AWOL but of those patients who failed to return to hospital after authorised leavie

There were 31 incidents of AWOL by 21 patients, 7 meif responsible for 10 incidents and 14 women for the otther 21. There was a predominance of younger people who were admitted by way of the police or on section. The mean age of these patients was 35 and 39.8 years respectively. Seventy-one per cent of the men and $33 \%$ of the women were admitted by way of the police or the Mental Health Act, $86 \%$ and $100 \%$ had previous admissions. Thirty-three per cent were diagnosed as suffering from manic depression, $24 \%$ schizophrenia, $14 \%$ depression, $10 \%$ personality disorder and $10 \%$ drug induced psychosis, figures comparable to other reports: Again, as expected, $50 \%$ of the men and $24 \%$ of the women absconded within the first week; $55 \%$ of all patients returned on the same day; $10 \%$ within a week; $6.5 \%$ ( 2 men) failed to return and were discharged in their absence.

Twenty-nine per cent of the men and $36 \%$ of the women were on section at the time of absconding.
The police were informed in $50 \%$ of cases involving men and $38 \%$ of cases involving women. Most patients $(35 \%)$ went home and $20 \%$ were found on hospital grounds. The police returned $34 \%$ of A.WOL cases.

Recording of these incidents in the medical and nursing notes were not adequate; $42 \%$ were not recorded in the medical notes. The nursing notes were not available in two incidents $(6.5 \%)$.

The nursing notes were poorly filed, often in the wrong order and the wrong place. The "missing patient check list" which should be recorded by the nursing staff in each incident was only completed in two cases out of $31(6.5 \%)$.

According to information given by the hospital managers there does not seem to be a relation between absconding and the number of nursing staff in the relevant shift. The number of nurses on various shifts ranged between three and seven. It is interesting that during the study period there were no incidents of absconding when there were seven nurses on a shift.

\section{Conclusion and recommendation}

The pattern of AWOL in New Cross Hospital seems similar to that reported by Tomison (1989). Although AWOL behaviour is difficult to predict, the history of the previous admission, single status, younger age, diagnosis of psychiatric illness or personality disorder are the best predictors. Patients are more likely to abscond early in their admission and to return home. Recording all these incidents needs to be improved greatly. More than half were not recorded in medical notes because either the Responsibie Medical Officer was not notified or he failed to act on the notification. The missing patient checklist should be filled in in each case.

\section{Referénce}

Tomison, A. R. (1989) Characteristics of psychiatric hospital abscohders. The British Journal of Psychiatry, 154, $368-371$. 